\title{
miR-520h Inhibits cell survival by targeting mTOR in gestational diabetes mellitus
}

\author{
Jie Wen and Xiaoxia Bai ${ }^{\varpi}$ \\ Department of Obstetrics, Women's Hospital School of Medicine Zhejiang University, Hangzhou City, Zhejiang Province, 310006, China
}

Gestational diabetes mellitus (GDM) is a type of diabetes that occurs during pregnancy due to abnormal maternal glucose metabolism. This study aimed to investigate the effect of miR-520h and its potential target gene on the progression of GDM. The blood samples were taken from healthy pregnant women and GDM patients. Human villous trophoblasts HTR-8/SVNEO cells were treated with 25 $\mathrm{mM}$ glucose and were considered as the GDM cell model. The miR-520h level was detected using qRT-PCR in the serum and GDM cell model. The correlation analysis between fasting blood-glucose (FBG) level and miR-520h expression was analyzed. The target relationship between miR-520h and mTOR was verified using dual luciferase reporter assay. HG-induced cells were transfected with miR520h mimic or miR-520h inhibitor and pCDNA3-mTOR vector or their NCs. Cell viability, apoptosis and mTOR expression level were detected using CCK-8, flow cytometry and western blotting, respectively. The results showed that the miR-520h serum level was up-regulated in the GDM patients' serum and GDM cell model, and was positively correlated with FBG of GDM patients. High glucose (HG) inhibited HTR-8/SVNEO cell viability and decreased mTOR expression, while it promoted apoptosis. Then, the effects of HG on HTR-8/SVNEO cells were reversed by miR-520h inhibitor. Moreover, mTOR was identified as a target gene downstream of miR-520h. The overexpression of mTOR alleviated miR-520h mimic-induced reduction in cell viability and enhancement in cell apoptosis in the GDM cell model. In conclusion, miR-520h could inhibit cell viability and promote cell apoptosis by regulating mTOR expression in the GDM cell model. Hence, miR-520h might be a potential and important marker for the diagnosis and treatment of GDM.

Keyword: gestational diabetes mellitus (GDM), miR-520h, mTOR, trophoblast, apoptosis

Received: 10 June, 2020; revised: 22 September, 2020; accepted: 23 November, 2020; available on-line: 23 February, 2021

घe-mail: qwehg6R@163.com

Abbreviations: GDM, gestational diabetes mellitus; miRNAs, MicroRNAs; PARP1, poly ADP-ribose polymerase; FBG, fasting bloodglucose

\section{INTRODUCTION}

Gestational diabetes mellitus (GDM) is a type of diabetes that develops during pregnancy due to abnormal maternal glucose metabolism (Barnes-Powell, 2007). GDM predisposes pregnant women to produce excessive amniotic fluid, resulting in an oversized fetus, stillbirth, and miscarriage (Barbour et al., 2007; Yuan, 2018). It can also cause neonatal respiratory distress syndrome (NRDS), hypocalcemia, polycythemia and other diseases (Barbour et al., 2007). In addition, GDM can even affect intelligence development in childhood and increase the risk of obesity and diabetes in adolescence (Barbour et al., 2007). Therefore, it is very important to study the early diagnosis, treatment, and pathogenesis of GDM. In recent years, it has been generally believed that the pathogenesis of GDM is a complex issue that is caused by multiple factors, such as inflammatory factors, insulin resistance, dysfunction of islet $\beta$-cells, genetics, and environment (Plows et al., 2018). Previous studies have shown that alterations at a molecular level during pregnancy may be an important direction to explore the effects of GDM on pregnancy ( $\mathrm{Su}$ et al., 2010; Wander et al., 2017).

MicroRNAs (miRNAs) are endogenous non-coding small molecule RNAs, which can actively participate in the process of cancer cell differentiation, neoplasia, and vascular proliferation (Di Leva et al., 2014). In recent years, many miRNAs, such as miR-126, miR-146a and miR-29, have been found to act on pancreatic islets and are strongly related to insulin secretion, inflammation and insulin resistance, and may contribute to the progression of diabetes and GDM (Filios \& Shalev 2015; Wander et al., 2017). Furthermore, miR-657 could regulate cell proliferation and polarization into the M1 phenotype of macrophage, thus participating in the development of GDM (Wang et al., 2019). MiR-520h is a cancer-promoting factor participating in a variety of cancers, such as breast cancer, pancreatic cancer, and cervical cancer (Su et al., 2010; Li et al., 2011; Chang et al., 2014). In particular, a study reported that the miR-520h level is increased in the serum of patients with GDM, suggesting that miR-520h might be related to the pathogenesis of GDM (Gillet et al., 2019). Moreover, miR-520 is also found to promote trophoblast apoptosis by inhibiting expression of the poly ADP-ribose polymerase (PARP1) (Dong et al., 2017). Dysfunctions of the trophoblast may be closely relevant to the complications of pregnancy, such as GDM, fetal growth restriction, and miscarriage (Wang et al., 2019). However, whether miR-520h affects the survival of trophoblast cells in GDM requires further study.

Therefore, the purpose of this study was to explore the effect of miR-520h on the survival of trophoblast cells in GDM and explore its possible regulatory mechanism through establishing high-glucose (HG)-induced trophoblast cell model in vitro, providing a new target for the treatment of GDM.

\section{MATERIALS AND METHODS}

\section{Sample collection}

From January 2019 to January 2020, pregnant women diagnosed with GDM at the Women's Hospital School 
of Medicine Zhejiang University were considered as the GDM group $(n=32)$. Healthy pregnant women with normal glucose tolerance were treated as the healthy group $(n=48)$. In the GDM group, the average age of patients was $32.71 \pm 5.26$ years old and the average gestational age was $28.33 \pm 2.81$ weeks. In the healthy group, the average age of pregnant women was $29.13 \pm 4.22$ years old and the average gestational age was $29.10 \pm 2.32$ weeks. None of the participants had history of liver disease, hypertension, kidney disease, etc.

Elbow venous blood was collected from all participants under fasting conditions in the early morning. The fasting blood-glucose (FBG) of GDM patients' blood samples was immediately tested, and the blood samples were centrifuged for serum collection. The miR-520h serum level was detected by quantitative real time polymerase chain reaction (qRT-PCR). In addition, correlation analysis between the FBG level and miR-520h expression in GDM patients was analyzed.

All experimental procedures and protocols were approved by the Medical Ethical Committee of Women's Hospital School of Medicine Zhejiang University. The informed consent from all patients was obtained.

\section{Cell culture and treatment}

Human villous trophoblast cell line HTR-8/SVNEO (Procell, China) was cultured in Roswell Park Memorial Institute (RPMI)-1640 medium (Solarbio, China) supplemented with 5\% fetal bovine serum (FBS, Solarbio) and $1 \%$ Penicillin-Streptomycin (P/S, Solarbio) at $37^{\circ} \mathrm{C}$.

Cultured cells were divided into two group: one group was treated with $25 \mathrm{mM}$ glucose and annotated as the HG group, the other group was treated with $5.5 \mathrm{mM}$ glucose and annotated as the control group.

Cells in the control or HG groups were transfected with $50 \mathrm{nM}$ miR-520h mimic or negative control (NC) mimic, $100 \mathrm{nM}$ miR-520h inhibitor or NC inhibitor, respectively, using Lipofectamine 3000 (Thermo Fisher Scientific, USA). In addition, cells in the HG group were also transfected with $50 \mathrm{nM}$ pCDNA3-mammalian target of rapamycin (pCDNA3-mTOR) vector, or co-transfected with miR-520h mimic and pCDNA3.1 vector or pCDNA3-mTOR vector, using Lipofectamine 3000 at $37^{\circ} \mathrm{C}$.

\section{qRT-PCR}

Total RNA from cells in the control or HG groups was extracted using QIAEX II Gel Extraction Kit (QIAGEN, Germany) and cDNA synthesis was performed using QuantiTect Transcription Kit (QIAGEN). The miR-520h expression level was detected using miScript miRNA PCR Arrays (QIAGEN). The primer sequences for miR-520h were 5'-ACAAAGUGCUUCCCUUUAGAGU-3' (forward) and 5'-UCUAAAGGGAAGCACUUUGUUU-3' (reverse). The primer sequence for $\mathrm{NC}$ mimic was 5'-CT'TCAAGTAATCCAGGATAGGG-3', and the primer sequence for $\mathrm{NC}$ inhibitor was 5'-CAGTACT'T'TGTGTAGTACAA-3'. The relative level of miR-520h was normalized to U6 and analyzed using the 2- $\Delta \Delta \mathrm{Ct}$ method (Livak \& Schmittgen 2001). The primer sequences for U6 were 5'-GCT'TCGGCAGCACATATACT-3' (forward), and 5'-GTGCAGGGTCCGAGGTATTC-3' (reverse).

\section{Cell viability}

Cells in the control or HG groups were co-incubated with the Cell Counting Kit-8 solution (CCK-8, Solar- bio) at $37^{\circ} \mathrm{C}$ for $2 \mathrm{~h}$ to detect cell viability. The optical density (OD) value at $450 \mathrm{~nm}$ was measured using an ultraviolet spectrophotometer (Shanghai Aucy Scientific Instrument, China).

\section{Cell apoptosis}

Cells in the control or HG groups were fixed and stained using the Hoechst Staining Kit (Beyotime, China), digested with $0.25 \%$ trypsin (Beyotime), and then 5 $\mu \mathrm{L}$ of Annexin-V and Propidium (PI) solution (Beyotime) were added to co-incubate for $10 \mathrm{~min}$. Differentiation of apoptotic cells (Annexin- $\mathrm{V}$ positive) was detected using flow cytometry (Beckman Coulter, USA).

\section{Dual luciferase reporter assay}

Cells were co-transfected with miR-520h mimic or NC mimic and mTOR wild type (WT) or mTOR mutant (MUT) using Lipofectamine 3000 (Thermo Fisher Scientific). Then, the relative luciferase activity in the cells was detected using Dual-Luciferase ${ }^{\circledR}$ Reporter Assay System Protocol (Promega, USA).

\section{Western blotting}

Cells in the control or HG groups were lysed to collect total protein. After protein transfer onto the nitrocellulose (NC) membrane, the membrane was placed in $5 \%$ milk for $2 \mathrm{~h}$ at $37^{\circ} \mathrm{C}$ and co-incubated with the mTOR (ab2732, Abcam, USA) and $\beta$-actin antibodies (ab179467, Abcam) that were diluted 1500 times, for $16 \mathrm{~h}$ at $4^{\circ} \mathrm{C}$. Then, the anti-rabbit IgG antibody (ab191866) conjugated with horseradish peroxidase was diluted 1500 times and co-incubated with NC membrane for $1 \mathrm{~h}$. The bands on the membrane were observed and recorded by using an imaging system (Bio$\mathrm{Rad}, \mathrm{USA}$ ) and analyzed using the Image $\mathrm{J}$ software (NIH Image, USA).

\section{Statistical analysis}

All of the experiments were repeated three times and analyzed using SPSS 21.0 software (SPSS Inc., USA) with $t$-test for two groups and analysis of variance (ANOVA) for multiple groups, and then displayed as mean \pm standard deviation (S.D.). If $p<0.05$, the data were statistically significant.

\section{RESULTS}

\section{miR-520h was up-regulated in the GDM patients' serum and GDM cell model}

In order to study the relationship between miR$520 \mathrm{~h}$ level and blood glucose, qRT-RCR was used to detect the serum level of miR-520h in GDM patients. The result showed that the miR-520h serum level in the GDM group was higher than that in the healthy group (Fig. 1A, $p<0.001$ ). Furthermore, the miR-520h serum level was positively correlated with the FBG level in GDM patients (Fig. 1B). In Fig. 1C, the results also show that miR-520h expression was significantly up-regulated in the HG-induced HTR-8/SVNEO cells when compared to the normal cells $(p<0.001)$. To sum up, these data demonstrated that miR-520h was up-regulated in GDM patients. 

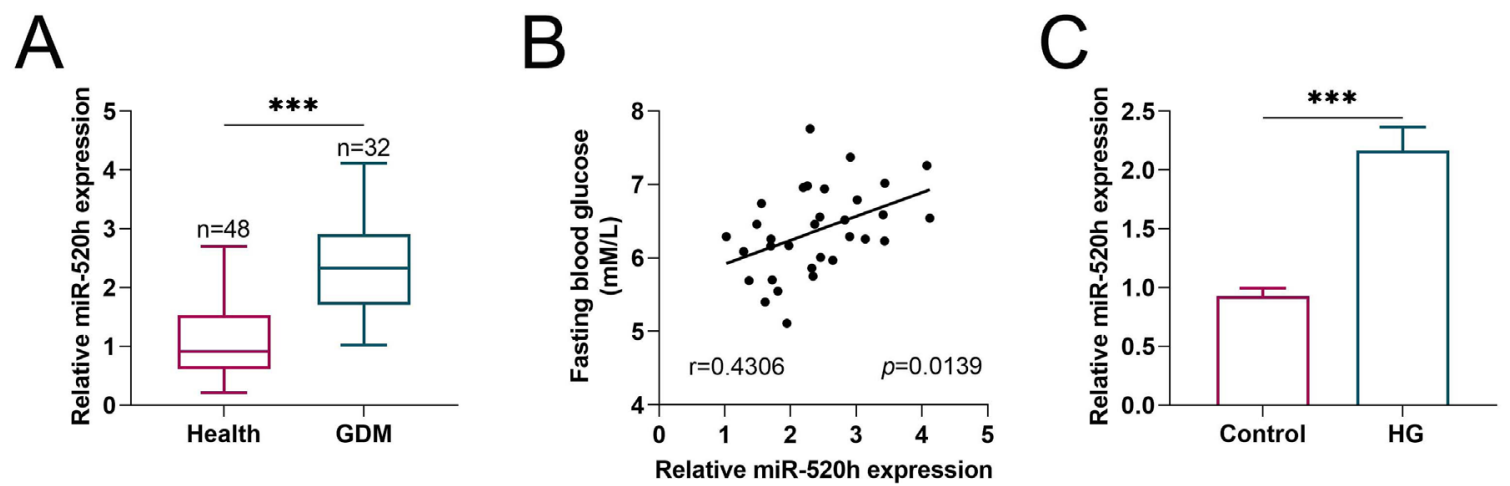

Figure 1. miR-520h is up-regulated in the GDM patients' serum and in the GDM cell model.

(A) The miR-520h serum level was detected using RT-qPCR in the serum from GDM patients and healthy pregnant women. (B) Correlation analysis of fasting blood-glucose (FBG) and miR-520h expression in GDM patients. (C) HTR-8/SVNEO cells in the HG group were treated with $25 \mathrm{mM}$ glucose, which was used as the GDM cell model. The relative miR-520h expression was detected using RT-qPCR. ${ }^{* * *} p<0.001$.

\section{miR-520h affected cell viability and apoptosis in the GDM cell model}

MiR-520h mimic or miR-520h inhibitor were respectively transfected into normal HTR-8/SVNEO cells and HG-induced HTR-8/SVNEO cells. The transfection efficiency of miR-520h mimic or inhibitor is shown in Fig. 2A and Fig. 2B, which demonstrate that miR-520h expression was up-regulated when cells were transfected with the miR-520h mimic, while it was down-regulated when cells were transfected with the miR-520h inhibitor $(p<0.001)$. CCK-8 results in Fig. 2C indicate that the viability of HG-induced HTR-8/SVNEO cells was decreased when compared to the control cells $(p<0.01)$. The miR-520h mimic significantly reduced the viability
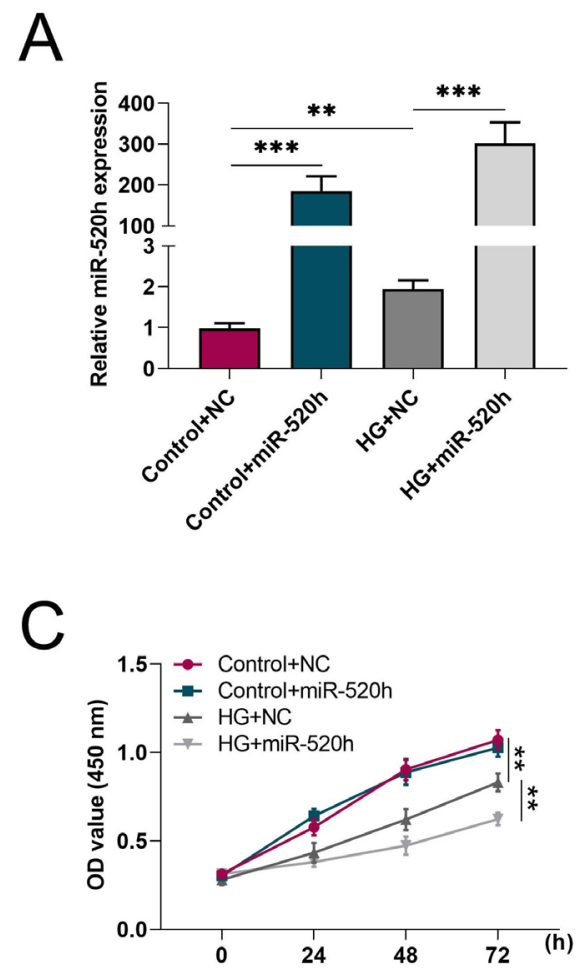

of HG-induced HTR-8/SVNEO cells $(p<0.01)$. In contrast, as shown in Fig. 2D, the viability of HG-induced HTR-8/SVNEO cells was increased after transfection with the miR-520h inhibitor $(p<0.01)$. In addition, the results from flow cytometry indicated that the apoptotic rate was significantly increased in the HG-induced HTR-8/SVNEO cells (Fig. 3, $p<0.001$ ). The miR-520h mimic had aggravated the enhancement of apoptotic rate induced by $\mathrm{HG}$, but the miR-520h inhibitor alleviated HG-induced increase of the apoptotic rate $(p<0.01)$. Hence, the above data illustrate that HG could inhibit viability and promote apoptosis of HG-induced HTR-8/ SVNEO cells, and the miR-520h inhibitor reversed these results.
B
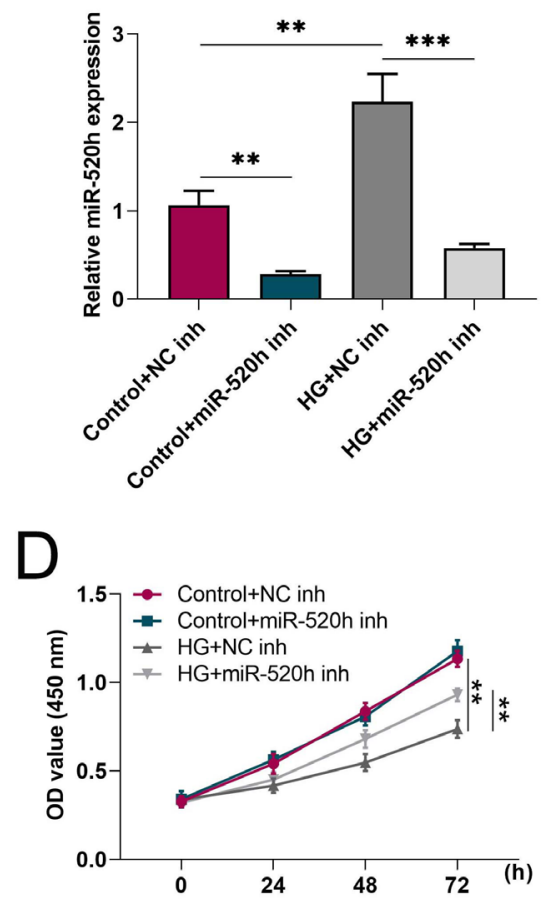

Figure 2. miR-520h inhibits the viability of HG-treated HTR-8/SVNEO cells.

The relative miR-520h expression was detected using RT-qPCR for control and HG-treated HTR-8/SVNEO cells transfected with (A) miR-520h mimic or NC-mimic, or (B) miR-520h inhibitor or NC inhibitor. Cell viability was detected using CCK-8 in the control and HG HG-treated HTR-8/SVNEO cells transfected with (C) miR-520h mimic or NC-mimic, or (D) miR-520h inhibitor or NC inhibitor. ${ }^{* *} p<0.01$; ${ }^{* * *} p<0.001$. 

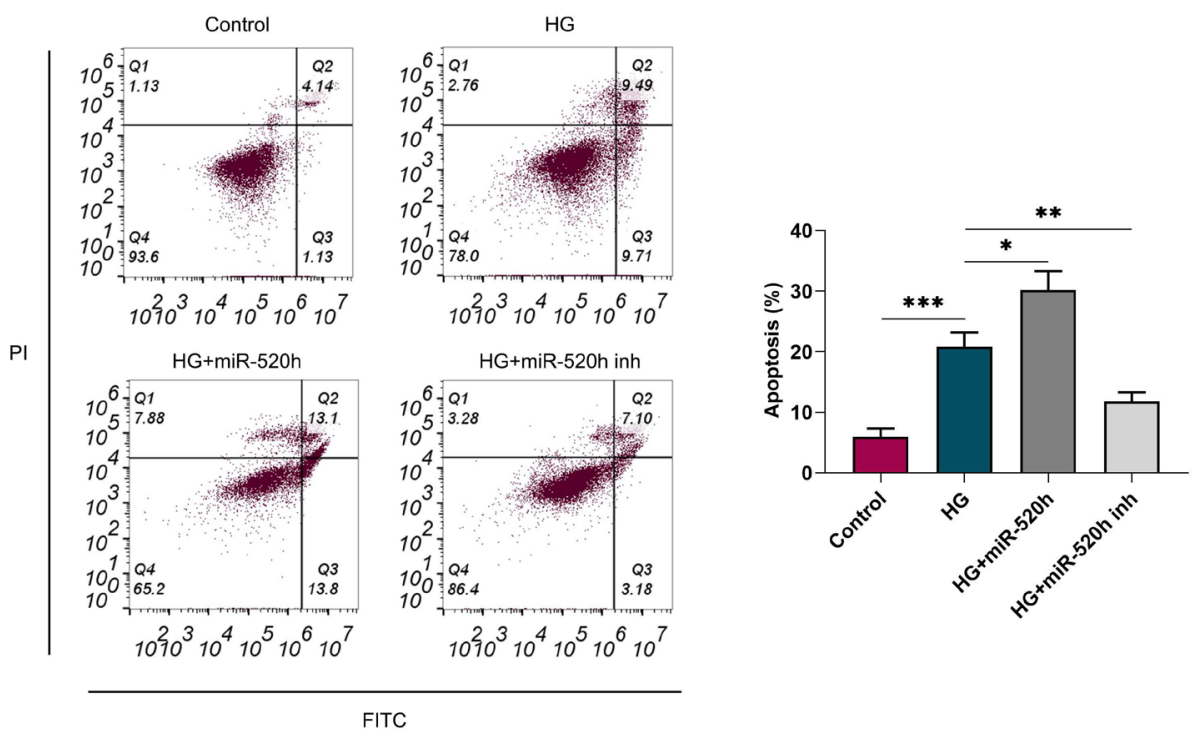

Figure 3. miR-520h promotes apoptosis of the HG-induced HTR-8/SVNEO cells.

miR-520h mimic or miR-520h inhibitor was transfected into HG-induced HTR-8/SVNEO cells. Apoptosis was detected by using flow cytometry. ${ }^{*} p<0.05 ;{ }^{* *} p<0.01 ;{ }^{* * *} p<0.001$.

\section{miR-520h targeted and regulated mTOR expression}

The predicted results obtained from Starbase (http:// starbase.sysu.edu.cn/index.php) revealed that mTOR might be a potential downstream target gene of miR-520h (Fig. 4A). Dual luciferase reporter assay in Fig. $4 \mathrm{~A}$ shows that the relative luciferase activity was reduced in the group that was co-transfected with mTOR-WT and miR-520h mimic when compared to the mTOR-WT and NC mimic group $(p<0.001)$. Meanwhile, there was no significant difference in the luciferase activity when cells were co-transfected with mTOR-MUT and miR-520h when compared to the mTOR- MUT and NC mimic group. These results verified that mTOR is a target gene of miR-520h. Furthermore, western blotting analysis showed that the protein level of mTOR was clearly suppressed in HG-induced HTR-8/SVNEO
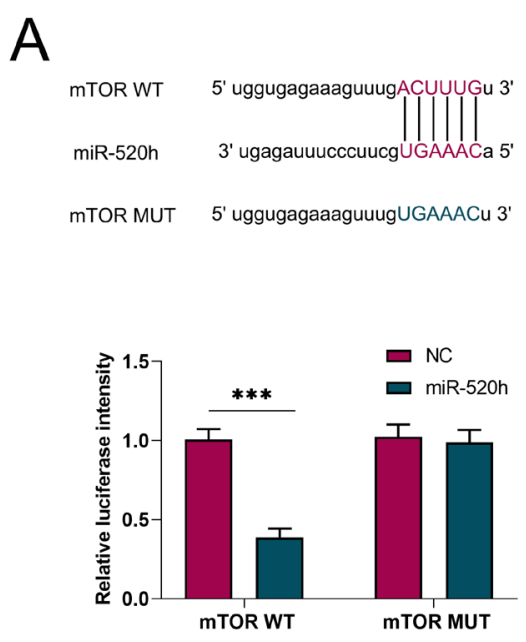

cells, which was reversed by the miR-520h inhibitor (Fig. $4 \mathrm{~B}, p<0.01)$. Therefore, these data demonstrated that miR-520h could target and regulate expression of mTOR in the GDM cell model.

\section{miR-520h affected cell viability and apoptosis by regulating $\mathrm{mTOR}$ expression in the GDM cell model}

Finally, miR-520h mimic and pCDNA3-mTOR vector were co-transfected into HG-induced HTR-8/SVNEO cells. As shown in Fig. 5A, the miR-520h mimic reduced mTOR expression, and then this reduction was recovered in the miR-520h mimic and pCDNA3-mTOR vector co-transfection group, illustrating that the miR-520h mimic and pCDNA3-mTOR vector were successfully cotransfected into cells $(p<0.001)$. The results presented in Fig. 5B and $5 \mathrm{C}$ indicate that the reduction in cell viabil-
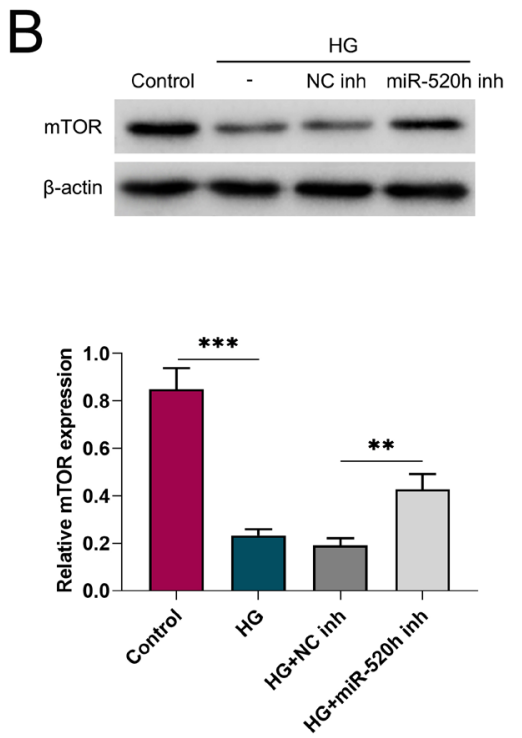

Figure 4. miR-520h targets and regulates mTOR expression.

(A) The target sequences between miR-520h and mTOR were predicted using Starbase (http://starbase.sysu.edu.cn/index.php), and the target relationship between miR-520h and mTOR was verified using dual luciferase reporter assay. (B) mTOR expression was detected using western blotting in HG-induced cells transfected with miR-520h inhibitor or NC inhibitor. ${ }^{* *} p<0.01 ;{ }^{* * *} p<0.001$. 

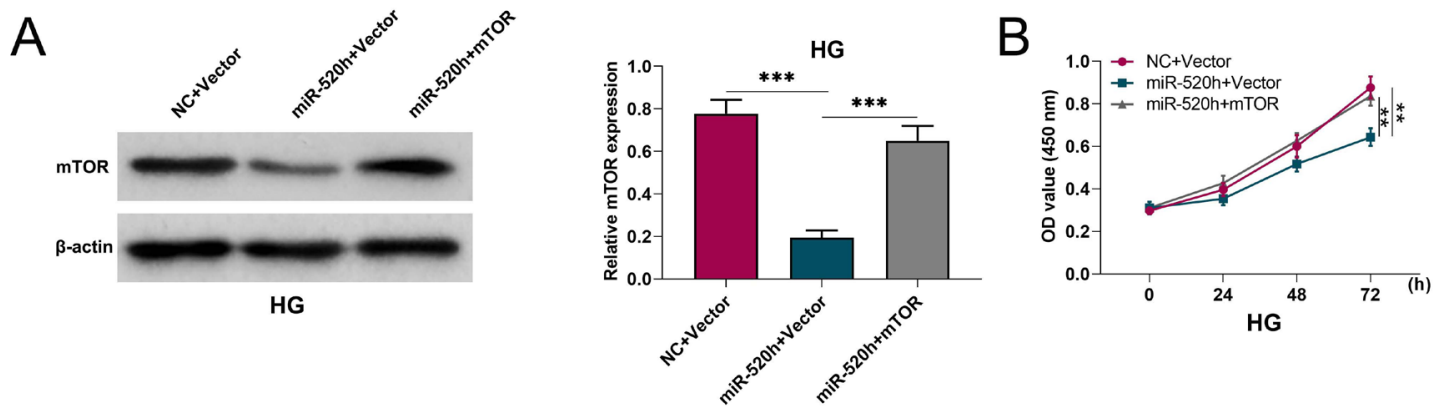

C
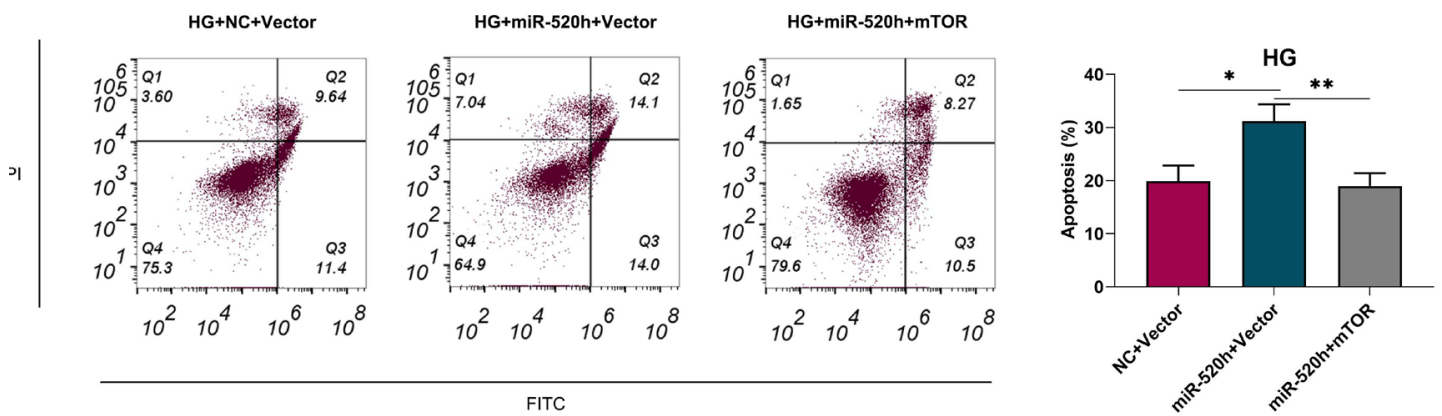

Figure 5. miR-520h affects the viability and apoptosis of HG-induced HTR-8/SVNEO cells by regulating mTOR.

HG-induced cells were co-transfected with NC mimic or miR-520h mimic and pCDNA3-mTOR vector or pCDNA3.1 vector. (A) mTOR expression was detected using western blotting. (B) Cell viability was detected using CCK-8. (C) Apoptosis was detected using flow cytometry. ${ }^{*} p<0.05 ;{ }^{* *} p<0.01 ;{ }^{* * *} p<0.001$.

ity and induction of apoptotic rate induced by miR-520h mimics were alleviated when mTOR was overexpressed in the HG- induced HTR-8/SVNEO cells $(p<0.05)$. The above results demonstrated that miR-520h inhibited viability and promoted apoptosis by targeting and regulating expression of m'TOR in the GDM cell model.

\section{DISCUSSION}

Some physiological changes that occur in women during pregnancy can lead to the increased requirements of glucose and a relative insufficiency of insulin secretion in their bodies, which finally cause GDM (Xie et al., 2000). As a very common disease, GDM can also affect fetal metabolism and development (Fornes et al., 2018).

MiRNAs have functions in regulating gene expression in the growth, development and disease of organisms, and are closely related to GDM, such as miR-503, miR-657 and miR-520h (Zhao et al., 2014; Wang et al., 2019). For instance, miR-503 expression is increased in the placental tissue and peripheral blood of patients with GDM (Xu et al., 2017). The level of miR-520h in the serum from GDM patients is also reported to be upregulated (Gillet et al., 2019). Consistently, the results of the study presented here show that the serum level of miR-520h in the patients with GDM was increased, indicating that miR-520h might be related to the progression of GDM. In addition, we found that the miR-520h level was positively correlated with FBG in GDM patients due to the occurrence of insulin resistance or reduced uptake and utilization levels of insulin-stimulated glucose (Godinjak et al., 2012). Therefore, this study illustrated that miR-520h was upregulated and was accompanied by an increase of blood glucose in GDM patients.

The placenta is an important place for maternal-fetal exchange of nutrients. The adhesion and invasion of placental trophoblast cells to the maternal endometrium is the prerequisite for the formation of the placenta (Wang et al., 2019). A study has found that abnormal trophoblast function may be closely relevant to the progression of GDM (Wang et al., 2019). Moreover, Dong et al., showed that miR-520 regulates trophoblast apoptosis by inhibiting expression of the poly ADP-ribose polymerase (PARP1) (Dong et al., 2017), inferring that the effect of miR-520h on GDM may be in the trophoblasts. Therefore, HTR-8/ SVNEO cells were treated with $25 \mathrm{mM}$ glucose to establish an in vitro GDM cell model in this study. The results showed that miR-520h was also up-regulated in the GDM cell model. Furthermore, Peng et al., found that miR-137 suppresses the survival and migration of HTR-8/SVNEO cells through down-regulating FNDC5 in GDM (Peng et al., 2019). Dong et al., reported that miR-520 inhibits PARP1 to promote trophoblast apoptosis (Dong et al., 2017). The study presented here has found that the effects of miR-520h on cell viability and apoptosis in the GDM cell model were like the results from the Dong's study, that is, miR-520h could inhibit cell viability and promote apoptosis in the GDM cell model. However, these results are in stark contrast to the role of miR-520h in a variety of cancers. Therefore, the function of miR-520h in GDM may differ from various diseases, and we suspect that the reason for these differences is that miR-520h targets some different genes in GDM.

mTOR can participate in multiple physiological activities in many cells, while it receives and integrates various stimuli inside and outside the cells. Both, HG and high insulin stimuli could promote phosphorylation of mTOR, causing changes in its downstream regulatory factors (Zoncu et al., 2011). In addition, a previous report revealed that miR-503 targets mTOR pathway to destroy the function of pancreatic islet $\beta$ cells to increase apoptosis in GDM (Xu et al., 2017). Therefore, mTOR is involved in pathogenesis of GDM. However, it is unknown whether miR-520h could regulate mTOR to participate in the progression of GDM. 
Thus, this is the first evidence discovering that mTOR might be a potential downstream target gene of miR-520h through bioinformatics. Moreover, mTOR's expression was down-regulated in the GDM cell model and had a negative correlation with miR-520h expression, and the luciferase reporter assay further confirmed the targeting relationship between miR-520h and mTOR. Therefore, we speculated that miR-520h affected GDM cell survival through targeting and regulating $\mathrm{m}$ TOR. The subsequent experimental results were indeed consistent with our speculation, showing that the reduction in cell viability and induction in cell apoptotic rate induced by miR-520h mimics were alleviated when mTOR was overexpressed in the GDM cell model. These results fully demonstrated that miR-520h could inhibit cell viability and promote apoptosis by regulating expression of m'TOR in the GDM cell model.

In conclusion, miR-520h could inhibit cell viability and promote apoptosis by regulating $\mathrm{mTOR}$ expression in GDM. Therefore, miR-520h may be an important marker of GDM, and has an application value in the diagnosis and treatment of GDM. However, more downstream target genes and regulatory pathway of miR-520h, as well as their specific mechanisms of action and effects on GDM cell growth or survival need to be further studied.

\section{Acknowledgements}

Not applicable.

\section{Funding}

Not applicable.

\section{Competing interests}

The authors state that there are no conflicts of interest to disclose.

\section{Ethics approval}

All experimental procedures and protocols were approved by the Medical Ethical Committee of Women's Hospital School of Medicine Zhejiang University.

\section{Statement of Informed Consent}

Written informed consent was obtained from a legally authorized representative(s) for anonymized patient information to be published in this article.

\section{Authors' contributions}

JW and $\mathrm{XB}$ designed the study, supervised the data collection, analyzed the data, interpreted the data and prepared the manuscript for publication, and reviewed the draft of the manuscript. All authors have read and approved the manuscript.

\section{Availability of data and materials}

All data generated or analyzed during this study are included in this published article.

\section{REFERENCES}

Barbour LA, McCurdy CE, Hernandez TL, Kirwan JP, Catalano PM, Friedman JE (2007) Cellular mechanisms for insulin resistance in normal pregnancy and gestational diabetes. Diabetes Care 30 (Suppl 2): S112-S119. https://doi.org/10.2337/dc07-s202

Barnes-Powell LL (2007) Infants of diabetic mothers: the effects of hyperglycemia on the fetus and neonate. Neonatal Netw 26: 283-290. https://doi.org/10.1891/0730-0832.26.5.283

Chang YW, Chen MW, Chiu CF, Hong CC, Cheng CC, Hsiao M, Chen CA, Wei LH, Su JL (2014) Arsenic trioxide inhibits CXCR4- mediated metastasis by interfering miR-520h/PP2A/NF- $x \mathrm{~B}$ signaling in cervical cancer. Ann Surg Oncol 21 (Suppl 4): S687-S695. https://doi.org/10.1245/s10434-014-3812-5

Di Leva G, Garofalo M, Croce CM (2014) MicroRNAs in cancer. Annu Rev Pathol 9: 287-314. https://doi.org/10.1146/annurevpathol-012513-104715

Dong X, Yang L, Wang H (2017) miR-520 promotes DNA-damageinduced trophoblast cell apoptosis by targeting PARP1 in recurrent spontaneous abortion (RSA). Gynecol Endocrinol 33: 274-278. https://doi.org/10.1080/09513590.2016.1266476

Filios SR, Shalev A (2015) $\beta$-Cell MicroRNAs: small but powerful. Diabetes 64: 3631-3644. https://doi.org/10.2337/db15-0831

Fornes D, White V, Higa R, Heinecke F, Capobianco E, Jawerbaum A (2018) Sex-dependent changes in lipid metabolism, PPAR pathways and microRNAs that target PPARs in the fetal liver of rats with gestational diabetes. Mol Cell Endocrinol 461: 12-21. https://doi. org/10.1016/j.mce.2017.08.004

Gillet V, Ouellet A, Stepanov Y, Rodosthenous RS, Croft EK, Brennan K, Abdelouahab N, Baccarelli A, Takser L (2019) miRNA Profiles in extracellular vesicles from serum early in pregnancies complicated by gestational diabetes mellitus. J Clin Endocrinol Metab 104: 5157-5169. https://doi.org/10.1210/jc.2018-02693

Godinjak A, Godinjak Z, Burekovic A, Surkovic I, Dizdarevic-Bostandzic A, Velija-Asimi Z (2012) Insulin resistance and lipid accumulation product in corelation to body mass index in women with polycystic ovary syndrome. Med Arch 66: 409-411. https://doi. org/10.5455/medarh.2012.66.409-411

Li X, Pan YZ, Seigel GM, Hu ZH, Huang M, Yu AM (2011) Breast cancer resistance protein BCRP/ABCG2 regulatory microRNAs (hsa-miR-328, -519c and $-520 \mathrm{~h}$ ) and their differential expression in stem-like ABCG2+ cancer cells. Biochem Pharmacol 81: 783-792. https://doi.org/10.1016/j.bcp.2010.12.018

Livak KJ, Schmittgen TD (2001) Analysis of relative gene expression data using real-time quantitative PCR and the 2(-Delta Delta C(T)) method. Methods 25: 402-408. https://doi.org/10.1006/ meth.2001.1262

Peng HY, Li MQ, Li HP (2019) MiR-137 Restricts the viability and migration of HTR-8/SVneo cells by downregulating FNDC5 in gestational diabetes mellitus. Curr Mol Med 19: 494-505. https://doi.org $/ 10.2174 / 1566524019666190520100422$

Plows JF, Stanley JL, Baker PN, Reynolds CM, Vickers MH (2018) The Pathophysiology of Gestational Diabetes Mellitus. Int J Mol Sci 19: https://doi.org/10.3390/ijms19113342

Su JL, Chen PB, Chen YH, Chen SC, Chang YW, Jan YH, Cheng $\mathrm{X}$, Hsiao M, Hung MC (2010) Downregulation of microRNA miR520h by E1A contributes to anticancer activity. Cancer Res 70: 50965108. https://doi.org/10.1158/0008-5472.Can-09-4148

Wander PL, Boyko EJ, Hevner K, Parikh VJ, Tadesse MG, Sorensen TK, Williams MA, Enquobahrie DA (2017) Circulating early- and mid-pregnancy microRNAs and risk of gestational diabetes. Diabetes Res Clin Pract 132: 1-9. https://doi.org/10.1016/j. diabres.2017.07.024

Wang P, Wang Z, Liu G, Jin C, Zhang Q, Man S, Wang Z (2019) miR-657 Promotes macrophage polarization toward M1 by targeting FAM46C in gestational diabetes mellitus. Mediators Inflamm 2019: 4851214. https://doi.org/10.1155/2019/4851214

Wang S, Sun F, Han M, Liu Y, Zou Q, Wang F, Tao Y, Li D, Du M, Li H, Zhu R (2019) Trophoblast-derived hyaluronan promotes the regulatory phenotype of decidual macrophages. Reproduction 157: 189-198. https://doi.org/10.1530/rep-18-0450

Xie R, Wang S, Wei L (2000) Insulin secretion and resistance during pregnancy in women with glucose intolerance. Zhonghua Fu Chan Ke Za Zhi 35: 709-711.

Xu K, Bian D, Hao L, Huang F, Xu M, Qin J, Liu Y (2017) microRNA-503 contribute to pancreatic beta cell dysfunction by targeting the mTOR pathway in gestational diabetes mellitus. Excli J 16: 1177-1187. https://doi.org/10.17179/excli2017-738

Yuan S-M (2018) Te application of ex utero intrapartum treatment (EXIT) procedure for cardiothoracic disorders. Signa Vitae: J Int Care Emerg Med 14: 14-16.

Zhao C, Zhang T, Shi Z, Ding H, Ling X (2014) MicroRNA-518d regulates PPAR $\alpha$ protein expression in the placentas of females with gestational diabetes mellitus. Mol Med Rep 9: 2085-2090. https:// doi.org/10.3892/mmr.2014.2058

Zoncu R, Efeyan A, Sabatini DM (2011) mTOR: from growth signal integration to cancer, diabetes and ageing. Nat Rev Mol Cell Biol 12: 21-35. https://doi.org/10.1038/nrm3025 